\title{
Lentivirus-mediated shRNA targeting of cyclin D1 enhances the chemosensitivity of human gastric cancer to 5-fluorouracil
}

\author{
JIN-HEE SEO ${ }^{1}$, EUI-SUK JEONG ${ }^{1}$, KYOUNG-SUN LEE ${ }^{1,2}$, SEUNG-HO HEO ${ }^{1,3}$, \\ DONG-GU JEONG ${ }^{1}$ and YANG-KYU CHOI ${ }^{1}$ \\ ${ }^{1}$ Department of Laboratory Animal Medicine, College of Veterinary Medicine, Konkuk University, Seoul 143-701; \\ ${ }^{2}$ Laboratory Animal Center, Osong Medical Innovation Foundation, Cheongwon-gun, Chungcheongbuk-do 363-954; \\ ${ }^{3}$ Asan Institute for Life Sciences, University of Ulsan College of Medicine, Seoul 138-736, Republic of Korea
}

Received June 28, 2013; Accepted August 5, 2013

DOI: $10.3892 /$ ijo.2013.2119

\begin{abstract}
Gastric cancer is one of the major public health problems. Despite new chemotherapeutic treatments, the prognosis of gastric cancer remains poor. 5-Fluorouracil (5-FU) is used as a standard chemotherapy drug in gastric cancer. However, 5-FU resistance develops frequently and is a main cause of chemotherapy failure in human gastric cancer. Overexpression of cyclin D1 is related to rapid cell growth, a poor prognosis and increased chemoresistance in several types of cancers. In this study, we investigated whether treatment of gastric cancer cells with shRNA targeting cyclin D1 (ShCCND1) or 5-FU, alone or in combination, influences the activation of phosphorylated AKT (pAKT) and pNFкB, which are markers that are increased in 5-FU chemoresistance. We also investigated the effect of combined treatment with ShCCND1 and 5-FU on cell growth and chemosensitivity to 5-FU in the gastric cancer cell line AGS. The data showed that ShCCND1-mediated cyclin D1 downregulation in AGS cells significantly inhibited cell proliferation, cell mobility and clonogenicity. In addition, combined treatment with ShCCND1 and 5-FU significantly decreased the survival rate of AGS cells, compared to single-treatment with either agent. These results demonstrated that ShCCND1 increases 5-FU chemosensitivity, a conclusion that is also supported by the concomitant reduction in expression of pAKT and $\mathrm{pNF} \kappa \mathrm{B}$, increase of G1 arrest and induction of apoptosis. Taken together, these data provide further evidence that therapeutic strategies targeting cyclin D1 may have the dual advantage of suppressing the growth of cancer cells, while enhancing their chemosensitivity.
\end{abstract}

Correspondence to: Dr Yang-Kyu Choi, Department of Laboratory Animal Medicine, College of Veterinary Medicine, Konkuk University, 1 Hwayang-dong, Gwangjin-gu, Seoul 143-701, Republic of Korea E-mail: yangkyuc@konkuk.ac.kr

Key words: gastric cancer, cyclin D1, 5-fluorouracil, shRNA

\section{Introduction}

Gastric cancer is one of the major public health problems and the main factor of cancer-related deaths in Eastern Europe and East Asia (1). Chemotherapy, including 5-FU, cisplatin and adriamycin, is a commonly used treatment method in gastric cancer (2). A significant survival advantage of 5-FU-based chemotherapy has been reported in patients with metastatic cancer as well as in those who have undergone surgery $(3,4)$. Although such treatments have increased the survival rate of gastric cancer patients, many patients treated with 5-FU-based chemotherapy have recurrence. Although resistance to 5-FU-based treatments is a major cause for recurrence, the mechanisms that drive the development of 5-FU resistance in cancer patients are poorly understood.

The cyclin D1 gene encodes for the proteins in the cyclindependent kinase (CDK)4/CDK6 complex. Cyclin D1 and CDK4/6 form a complex that phosphorylates and inactivates retinoblastoma $(\mathrm{RB})$ protein, a tumor suppressor $(5)$. The phosphorylation of RB results in the release of E2Fs, which then continues to activate genes that are necessary for advancing into the G1/S phases of the cell cycle (6). In accordance with its growth-promoting role, cyclin D1 can behave as an oncogene. Indeed, overexpression and/or rearrangement of the cyclin D1 gene is seen in several types of human cancers, including gastric cancer $(7,8)$. Therefore, suppression of cyclin D1 expression with a specific targeting method may serve as a powerful treatment for human gastric cancers and can be achieved via RNA interference (RNAi), one of the most effective methodologies for gene targeting.

Previous studies have demonstrated that overexpression of cyclin D1 increases resistance to radiotherapy or chemotherapeutic drugs in various cancer cells (9-13). Moreover, downregulation of cyclin D1 is related to induction of apoptosis and chemosensitivity in TTn cells (14). However, downregulation of cyclin D1 induces chemosensitivity to cis-diamminedichloroplatinum in human oral squamous cell carcinoma (15). The disparate effects of cyclin D1 downregulation may arise from the differential responses to the different drugs and cell type-specific effects, among other reasons. Therefore, cyclin D1 appears to act not only as a pro-survival factor but also as a pro-apoptotic factor depending on specifics 
of the experiment, such as the cell type and chemotherapy drug (16). AKT is a crucial molecule in protecting against cellular apoptosis and it plays a pivotal function in the regulation of normal cell growth and proliferation $(17,18)$. Previous studies have reported that the expression of AKT is changed in several human cancers and that this dysregulated expression may contribute to chemoresistance (19-21). Therefore, AKT is an attractive target of strategies aimed at overcoming chemoresistance in cancers.

The NFKB pathway is one of the main anti-apoptotic signaling pathways and is aberrantly activated in many cancer cells. Previous studies have described that some chemotherapeutic drugs, including 5-FU, can activate NFKB and, consequently, markedly suppress apoptosis $(22,23)$. Therefore, NFKB is closely linked to 5-FU chemoresistance in many cancer cells (24).

The aim of this study was to develop a lentivirus-mediated shRNA expression system targeting cyclin D1 to generate a stable silencing effect of sufficient efficiency for delivery of cyclin D1-specific shRNA (ShCCND1). We also sought to examine whether treatment with ShCCND1 or 5-FU, alone or in combination, influences the activation of phosphorylated AKT (pAKT) and $\mathrm{pNF \kappa B}$ and to determine the effect of the combined treatment with ShCCDN1 and 5-FU on cell growth and chemosensitivity to 5-FU in AGS cells.

\section{Materials and methods}

Cell line and chemicals. AGS gastric carcinoma cells were purchased from the Korean Cell Line Bank (KCLB, Seoul, Korea). Cells were maintained in RPMI-1640 medium with $10 \%$ fetal bovine serum and $1 \%$ penicillin/streptomycin at $37^{\circ} \mathrm{C}$ in an atmosphere of $5 \% \mathrm{CO}_{2}$ in a humidified chamber. The 293TN human embryonic kidney cells were purchased from System Biosciences (SBI, Mountain View, CA, USA) and maintained in high-glucose Dulbecco's modified Eagle's medium supplemented with $10 \%$ fetal bovine serum, glutamax and $1 \%$ penicillin/streptomycin at $37^{\circ} \mathrm{C}$ in an atmosphere of $5 \% \mathrm{CO}_{2}$ within a humidified chamber. The anticancer agent 5-FU was purchased from Sigma-Aldrich (St. Louis, MO, USA).

ShRNA-expressing plasmid DNA. The lentiviral expression vector (pGreenPuro ${ }^{\mathrm{TM}}$ vector) was purchased from SBI. Three different targeted sequences were designed to be homologous to the cyclin D1 gene (CCND1; GeneBank NM_053056). Target sites in human $C C N D 1$ were as follows: site 1, 5'-GCCC TCGGTGTCCTACTTCAAAT-3'; site 2, 5'-GCACGATTTCA TTGAACACTTCC-3'; and site 3, 5'-GGAAGTGTTCAATGA AATCGTGC-3'. These sequences were followed by a 12-bp 'loop' (CTTCCTGTCAGA) and the inverted repeat. These primer pairs were annealed and inserted into the BamHI and EcoRI sites of the pGreenPuro ${ }^{\mathrm{TM}}$ vector (25). In addition, a negative control shRNA sequence (ShScramble), which had nohomology tohumangenes(GACTTCATAAGGCGCATGC), was also designed using the same process described above (26). These plasmid DNAs were transformed into the E. coli DH5 $\alpha$ competent cells and purified using an endotoxin-free plasmid purification kit (Qiagen, Valencia, CA, USA). Successful ligation was confirmed by PCR and sequencing analyses. The resulting plasmids containing the scrambled and cyclin D1-specific shRNA sequences are hereafter referred to as pScramble and pCCND1, respectively.

Lentivirus generation and infection. 293TN cells were plated on $10-\mathrm{cm}$ culture plates at a density of $3 \times 10^{6}$ cells per plate. After a 24-h incubation, the cells were co-transfected with pScramble or pCCND1 and a lentiviral expression construct using Lipofectamine (Invitrogen, Grand Island, NY, USA). After $48 \mathrm{~h}$, the supernatant was collected and cleared through a $0.45-\mu \mathrm{m}$ filter. PEG-it ${ }^{\mathrm{TM}}$ virus precipitation solution was added to the clarified supernatant and the mixture was centrifuged at $1,500 \mathrm{x} \mathrm{g}$ for $30 \mathrm{~min}$ at $4^{\circ} \mathrm{C}$. The pellet was resuspended in 1/100th of the original volume of RPMI-1640 medium. One day prior to transduction, AGS cells were seeded at a density of $3 \times 10^{4}$ cells/well in 24 -well plates. After $24 \mathrm{~h}$, the culture medium was replaced by RPMI-1640 containing hexadimethrine bromide (5 $\mu \mathrm{g} / \mathrm{ml}$; Sigma-Aldrich). Then, AGS cells were infected with the pseudovirus stock. From the next day onward, AGS cells were maintained in RPMI-1640 medium containing puromycin $(1 \mu \mathrm{g} / \mathrm{ml})$. The stable puromycin-resistant cancer cell lines containing pScramble or pCCND1 obtained by subcloning on the selective medium were named as ShScramble and ShCCND1, respectively. The transduction efficiency of the stable AGS cells expressing shRNA was measured by flow cytometry.

Western blot analysis. AGS cells were lysed in radioimmunoprecipitation assay buffer (Sigma-Aldrich) containing a protease inhibitor cocktail (Sigma-Aldrich). AGS cells (ShScramble and ShCCND1) were centrifuged at 13,000 rpm for $10 \mathrm{~min}$. The protein concentration of the cell extract was determined using the $\mathrm{BCA}^{\mathrm{TM}}$ Protein Assay kit (Thermo Scientific, Rockford, IL, USA). The protein samples were boiled in sodium dodecyl sulfate-polyacrylamide gel electrophoresis (SDS-PAGE) loading buffer and then electrophoresed on $10 \%$ SDS-polyacrylamide gels. The proteins were transferred onto nitrocellulose membranes and the membranes were blocked with 5\% non-fat dry milk and incubated overnight with antibodies against cyclin D1 (Santa-Cruz Biotechnology, Santa Cruz, CA, USA), pRB (Cell Signaling Technology, Beverly, MA, USA), pAKT (Cell Signaling Technology), pNFkB (Cell Signaling Technology) and $\beta$-actin (Santa-Cruz Biotechnology) at $4^{\circ} \mathrm{C}$. Subsequently, the membranes were incubated with either anti-rabbit or anti-mouse secondary antibodies (Santa-Cruz Biotechnology). Specific antibodyprotein complexes were detected with the ECL Test kit (KPL, Gaithersburg, MD, USA). Densitometric analysis was performed using ImageJ software.

Cell proliferation and colony formation assay. Cell proliferation was analyzed using the Cell Counting kit-8 (CCK-8; Dojindo Laboratories, Kumamoto, Japan). ShScramble and ShCCND1 cells were plated on 96-well plates at a density of $10^{4}$ cells/well and cultured for $24 \mathrm{~h}$. Next, $10 \mu \mathrm{l}$ of CCK-8 solution was added to each well. After a 2-h incubation, an enzyme-linked immunosorbent assay (Tecan Sunrise, Sunnyvale, CA, USA) was performed and the absorbance of the samples was measured at $450 \mathrm{~nm}$.

To assess the colony formation ability, AGS cells containing shRNA were seeded (500 cells/well) in 6-well 
plates. Cell culture medium was replaced fresh RPMI-1640 every 2 days. After 3 weeks, cells were stained with $1 \%$ crystal violet for $30 \mathrm{~min}$. To identify colonies, the medium was removed and $3.7 \%$ formaldehyde was added. Subsequently, the number of colonies in each well was determined by counting the stained colonies under a light microscope. Image analysis was conducted using Metamorph version 7.5.6.0 software (Molecular Devices, CA, USA).

Scratch wound-healing assay. AGS cells were seeded on $60-\mathrm{mm}$ plates. When cells were $>90 \%$ confluent, a scratch was made using the tip of a pipette (27). After incubation for $48 \mathrm{~h}$, cells that were protruding from the border of the wound were observed and photographed using a Zeiss Axiovert 200 inverted microscope (Carl Zeiss MicroImaging, Thornwood, NY, USA) equipped with a 10x objective. Image analysis was conducted using Metamorph version 7.5.6.0 software.

In vitro cellular chemosensitivity to $5-F U$. To evaluate the cytotoxic effect of different concentrations of 5-FU, cells were seeded in 96-well plates at a density of $2 \times 10^{4}$ cells/well. After overnight incubation, the culture medium was replaced with fresh medium containing various concentrations of 5-FU $(0.1,1,5$ and $15 \mu \mathrm{g} / \mathrm{ml})$; a control set was included in which fresh medium alone was added, without any 5-FU. Cell viability was evaluated with the CCK- 8 assay $72 \mathrm{~h}$ after exposure to 5-FU.

To evaluate the cytotoxic effect of the combined treatment with ShCCND1 and 5-FU, a time-course cell viability assay was performed using the CCK-8 kit. Cells were seeded in 96-well plates at a density of $2 \times 10^{4}$ cells/well. After overnight incubation, the cells were treated with 5-FU $(15 \mu \mathrm{g} / \mathrm{ml})$. Cell viability was evaluated with a CCK-8 assay after 24, 48 and $72 \mathrm{~h}$.

Analysis of cell cycle and apoptosis. To analyze the cell cycle status, $10^{6}$ AGS cells were washed and resuspended in phosphate-buffered saline containing $5 \mu \mathrm{l}$ of RNase A $(10 \mathrm{mg}$ / $\mathrm{ml}$; Sigma-Aldrich) and $10 \mu \mathrm{l}$ of propidium iodide (PI; $1 \mathrm{mg} /$ $\mathrm{ml}$; Sigma-Aldrich). After incubating the samples for $1 \mathrm{~h}$ in the dark, the cell cycle distribution was assessed using a fluorescence-associated cell-sorting (FACS) assay with FACSCalibur (Becton-Dickinson, Rutherford, NJ, USA) and the results were analyzed with the CellQuest software (Becton-Dickinson).

To evaluate apoptosis, AGS cells were suspended in binding buffer at a cell density of $\sim 10^{6}$ cells $/ \mathrm{ml}$. Aliquots $(100 \mu \mathrm{l})$ of this cell suspension were incubated with Annexin$\mathrm{V}$-allophycocyanin and propidium iodide (BD Biosciences Pharmingen). After incubation, the samples were mixed with binding solution and analyzed on a flow cytometer (FACSCalibur, Becton-Dickinson). The data were analyzed using the CellQuest software (Becton-Dickinson).

Statistical analysis. For statistical analysis, all data obtained were analyzed using the Prism 5 software for Windows (GraphPad Software, San Diego, CA, USA). Statistically significant differences between the various groups were evaluated using the unpaired Student's t-test and Fisher's exact test. The level of statistical significance was set at values of $\mathrm{P}<0.05$.

\section{Results}

ShCCND1 inhibits the expression of cyclin D1 and cell proliferation in AGS cells. AGS cells stably transduced with lentivirus were successfully generated by selection on puromycin-containing medium. The transduction efficiency of the lentivirus-transduced AGS cells, as measured by flow cytometry, was 95.1 and $95.8 \%$ for the ShScramble and ShCCND1 cells, respectively (data not shown). The protein levels of cyclin D1 in AGS cells were examined by western blotting. Compared to the AGS cells, ShScramble expressed similar levels of cyclin D1 (data not shown). As shown in Fig. 1A, cyclin D1 expression levels were similar in ShCCND1_1- and ShScramble-transduced cells, whereas ShCCND1_2- and ShCCND1_3-transduced cells had decreased cyclin D1 levels compared to AGS cells (Fig. 1A). Densitometric analysis of the western blotting images indicated a dramatic and significant decrease in cyclin D1 expression in ShCCND1_3-transduced cells, compared to the parental AGS cells $(\mathrm{P}<0.05)$. The ShCCND1_3-transduced clone was then used for subsequent studies and named as ShCCND1. To evaluate the inhibitory effect of cyclin D1 knockdown on cancer cell proliferation, the cell viability of ShScramble- and ShCCND1-transduced cells was measured using the CCK- 8 assay. The viability of the ShCCND1-transduced cells was significantly diminished compared to that of ShScramble cells (Fig. 1B). Proliferation of these cells was considerably suppressed by ShCCND1transduced cells after day 1 of culture. These data suggested that ShCCND1 could effectively knockdown the endogenous cyclin D1 expression and inhibit cell proliferation in AGS cells.

ShCCNDl inhibits focus formation and cell motility in AGS cells. To test whether the knockdown of cyclin D1 affects the clonogenic potential, which is one of the important characteristics required for tumor formation in vivo, a colony formation assay was performed, as described previously (28). The number of foci in ShCCND1-transduced cells $(\mathrm{N}=12.7)$ was lower than that in ShScramble-transduced cells $(\mathrm{N}=63.7$, $\mathrm{P}<0.001$; Fig. 2A). Moreover, because decreased clonogenic potential is usually associated with invasive ability in cancer cells, the cell motility of AGS cells was analyzed using a classic wound-healing assay (28). The wound area in the ShCCND1transduced cells was significantly decreased compared to that in ShScramble-transduced cells (Fig. 2B); the wound area was 31.3 and $14.6 \%$ in ShCCND1- and ShScramble-transduced cells, respectively $(\mathrm{P}<0.001)$. These results demonstrated that ShCCND1 significantly decreased the focus-formation potential of the cells, which correlates with the formation of cancer in nude mice and their migratory capacity (29).

Combined treatment with ShCCNDI and 5-FU inhibits cell growth to a greater extent than treatment with either agent alone. High expression of cyclin D1 protein has been associated with increased resistance of cancer cells to chemotherapeutic agents, such as 5-FU $(12,13)$. Therefore, we investigated whether ShCCND1 could enhance the sensitivity of AGS cells to 5-FU. As shown in Fig. 3A, 5-FU treatment $(0.1,1$ and $5 \mu \mathrm{g} /$ $\mathrm{ml}$ ) decreased cell survival in a dose-dependent manner in AGS and in ShScramble- and ShCCND1-transduced cells. However, treatment with a high dose of 5-FU $(15 \mu \mathrm{g} / \mathrm{ml})$ decreased cell 
A

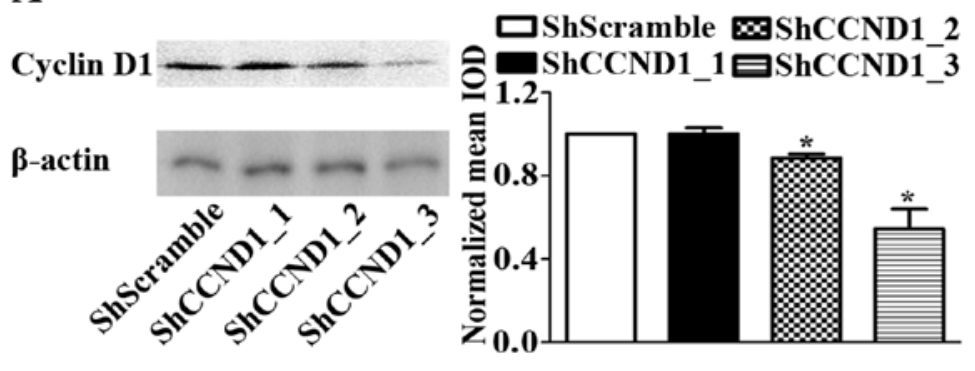

B

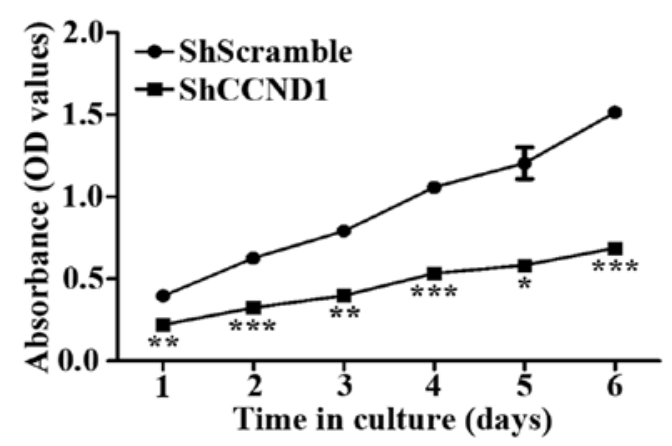

Figure 1. Expression of cyclin D1 and cell proliferation in AGS cells. (A) The levels of cyclin D1 in ShCCND1_2- and ShCCND1_3-transduced AGS cells was significantly inhibited, as indicated by western blot analysis. (B) ShCCND1 suppressed the growth of AGS cells compared to that of cells treated with ShScramble, as determined using the CCK-8 assay. The viability of the ShCCND1-transduced cells was significantly inhibited, compared to that of the ShScramble-treated cells. Data are presented as mean \pm SEM. ${ }^{*} \mathrm{P}<0.05,{ }^{* * *} \mathrm{P}<0.01$ and ${ }^{* * *} \mathrm{P}<0.001$ versus the ShScramble-transduced cells.

\section{A}
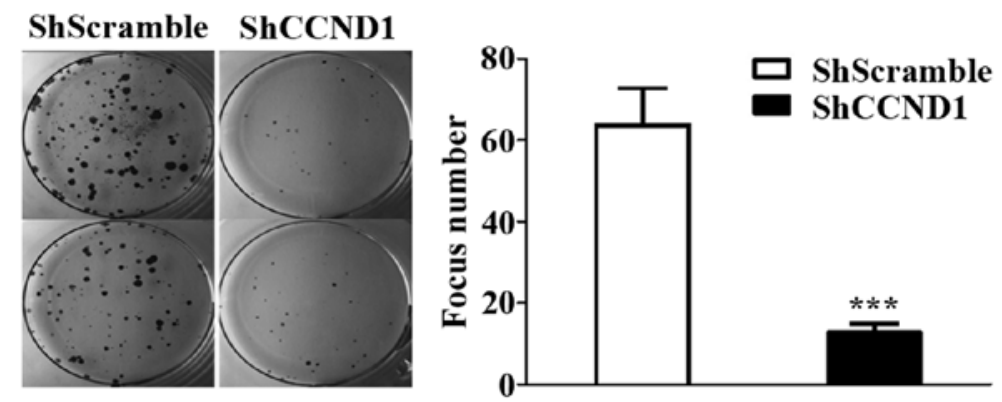

B

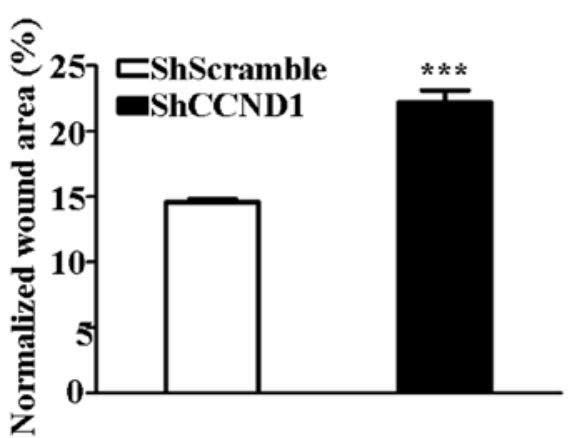

Figure 2. Colony formation and scratch wound-healing assays. (A) Colony formation assays of ShCCND1-transduced cells indicated the formation of a lower number of colonies compared to that in ShScramble-transduced. (B) The wound area of ShCCND1-transduced cells was significantly greater than that in ShScramble-transduced cells, as measured by scratch wound-healing assay. Data are presented as mean $\pm \mathrm{SEM} .{ }^{* * * *} \mathrm{P}<0.001$ versus the ShScramble-transduced cells.

survival in ShCCND1-transduced cells $(\mathrm{P}<0.05)$, but not in AGS and ShScramble-transduced cells. As shown in Fig. 3B (left panel), cell viability gradually increased in AGS and
ShScramble- and ShCCND1-transduced cells during culture without 5-FU. Compared to AGS and ShScramble-transduced cells, ShCCND1 cells exhibited significantly decreased cell 


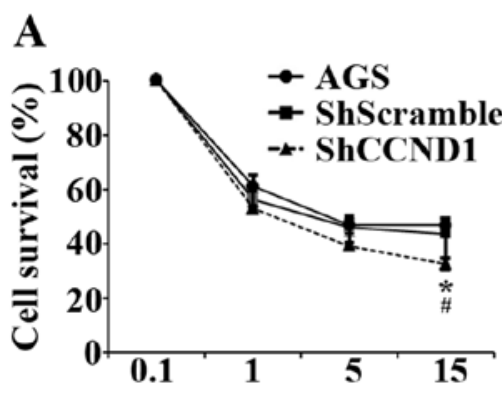

5-FU concentration $(\mu \mathrm{g} / \mathrm{ml})$

B

Control

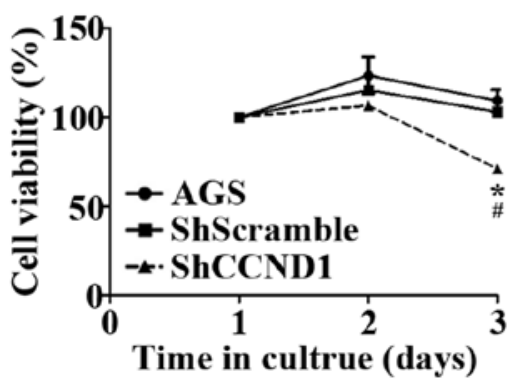

$5-\mathrm{FU} 15 \mu \mathrm{g} / \mathrm{ml}$

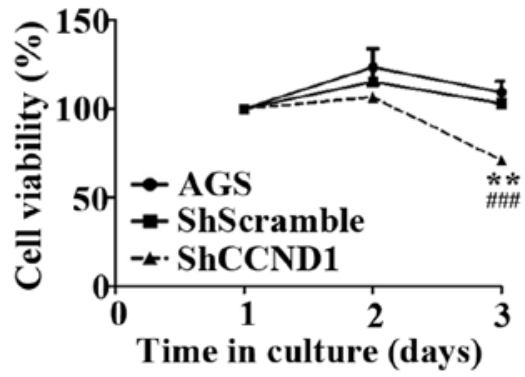

Figure 3. Effect of ShCCND1 and/or 5-FU on AGS cell viability, as measured by the CCK-8 assay. (A) ShCCND1-transduced cells alone exhibited a dosedependent decrease in cell survival after 5-FU treatment. (B) Time-course of the effect of ShCCND1 and/or 5-FU on AGS cell viability. Data are presented as mean \pm SEM. ${ }^{*} \mathrm{P}<0.05$ and ${ }^{* *} \mathrm{P}<0.01$, versus untreated parental AGS cells. ${ }^{\#} \mathrm{P}<0.05$ and ${ }^{\# \# \#} \mathrm{P}<0.001$, versus ShScramble-transduced cells.

viability on day $3(\mathrm{P}<0.05)$. These results reconfirmed that ShCCND1 inhibited the proliferation of AGS cells. Under the 5 -FU $(15 \mu \mathrm{g} / \mathrm{ml})$ treatment, the viability of ShCCND1transduced cells was significantly decreased compared to that of AGS cells $(\mathrm{P}<0.01)$ on day 2 and compared to AGS $(\mathrm{P}<0.01)$ and ShScramble-transduced $(\mathrm{P}<0.001)$ cells on day 3 (Fig. $3 \mathrm{~B}$ right panel). These findings indicate that combined treatments with 5-FU and ShCCND1 more effectively inhibited cell growth than treatment with 5-FU or ShCCND1 alone. These results indicated that ShCCND1 increases the sensitivity of AGS cells to 5-FU.

Combined treatment with ShCCNDI and 5-FU affects apoptosis and cell cycle regulation. To determine the potential effects of ShCCND1 on the cell cycle, ShCCND1-transduced cells were analyzed by flow cytometry. As shown in Fig. 4A, the G1-phase distribution was 76.7, 74.9 and $87.9 \%$ in cells treated with 5-FU, ShCCND1 and a combination of both agents, respectively. The percentage of cells arrested in the G1 phase in the combination-treated cells was much higher than that in ShScramble-transduced $(\mathrm{P}<0.001)$, ShCCND1treated $(\mathrm{P}<0.001)$ and 5-FU-treated cells $(\mathrm{P}<0.05)$. This increase was accompanied with a concomitant reduction in the percentage of cells in the S and G2 phases of the cell cycle. These data indicated that G1 phase arrest is induced in ShCCND1-transduced cells and that the combined treatment with ShCCND1 and 5-FU elicits a synergistic increase in G1 phase arrest, compared to treatment with ShCCND1 or 5-FU as single agents. In addition to cell cycle arrest, apoptotic cell death was measured by flow cytometry to assess the effect of the combined treatment on cell death. As shown in Fig. 4B, cellular apoptotic indices were $13.02 \%$ in cells without treat-
$\mathbf{A}$

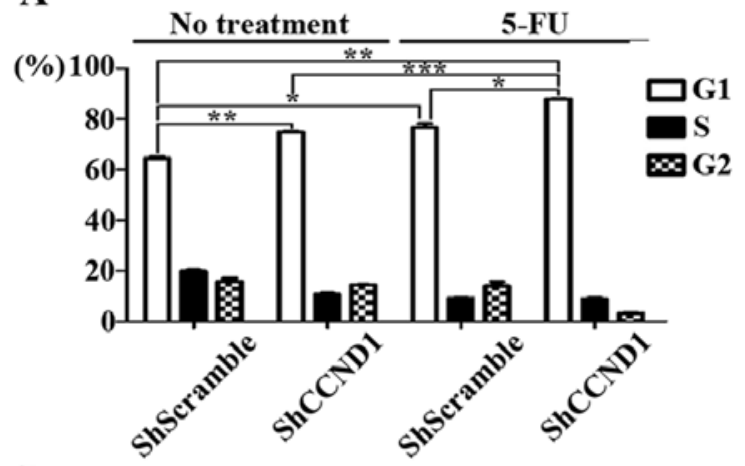

B

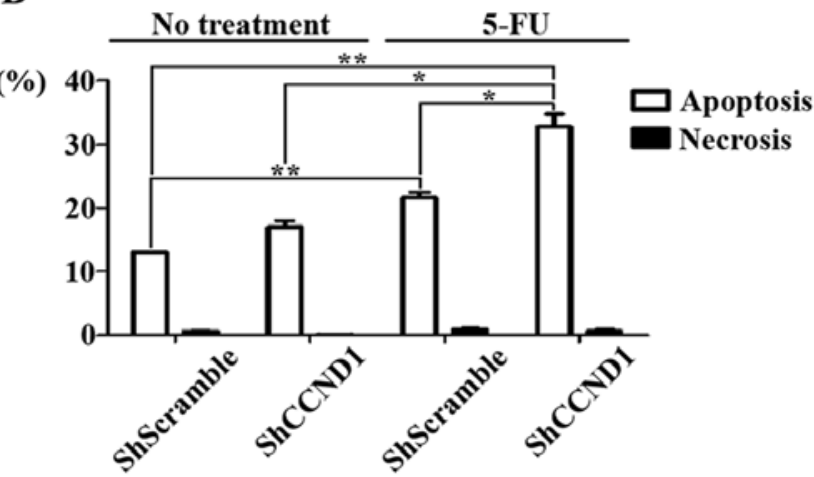

Figure 4. Effect of ShCCND1 and/or 5-FU on cell cycle distribution and apoptosis in AGS cells, as determined by flow cytometry. (A) Combined treatment with 5-FU and ShCCND1 significantly increased the proportion of cells in the G1 phase of the cell cycle, compared to the ShScramble-, ShCCND1-, or 5-FU-treated cells. (B) Apoptosis significantly increased in cells treated with the combination of ShCCND1 and 5-FU, compared to the ShScramble-, ShCCND1-, or 5-FU-treated cells. Data are presented as mean \pm SEM. ${ }^{*} \mathrm{P}<0.05,{ }^{* *} \mathrm{P}<0.01$ and ${ }^{* * *} \mathrm{P}<0.001$. 

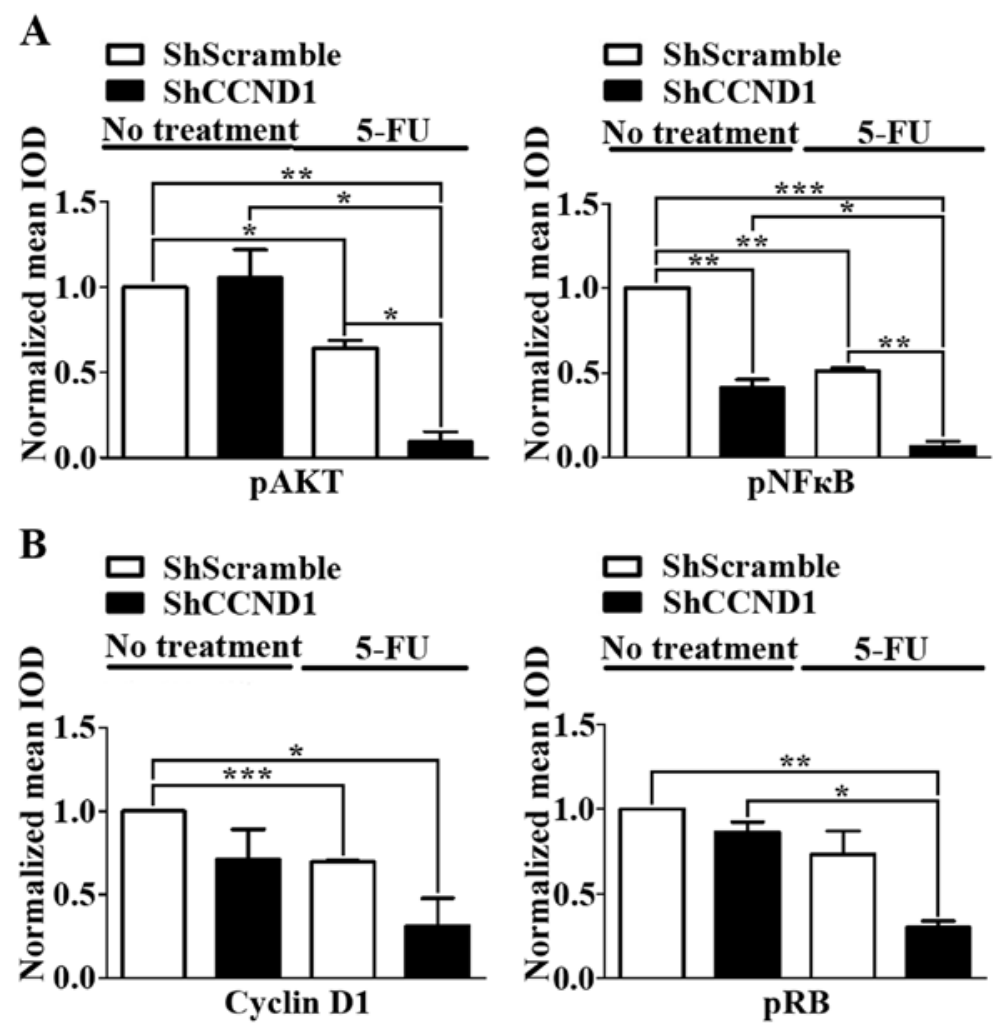

Figure 5. Effect of the treatment with ShCCND1 and/or 5-FU on the expression of pAKT, pNFkB and downstream signaling molecules. (A) Combined treatment with 5-FU and ShCCND1 significantly reduced pAKT and pNFkB expression compared to that in ShScramble-, ShCCND1-, or 5-FU-treated cells. (B) Combined treatment significantly reduced cyclin D1 and pRB expression compared to that in ShScramble-, ShCCND1-, or 5-FU-treated cells. Data are presented as mean \pm SEM. ${ }^{*} \mathrm{P}<0.05,{ }^{* *} \mathrm{P}<0.01$ and ${ }^{* * *} \mathrm{P}<0.001$.

ment, $17.03 \%$ in cells treated with ShCCND1 alone, $21.62 \%$ in cells treated with 5 -FU alone and $32.85 \%$ in cells treated with the combination. These results show that ShCCND1 in combination with 5-FU leads to significantly increased apoptosis, compared to that in untreated $(\mathrm{P}<0.05)$, ShCCND1- $(\mathrm{P}<0.05)$ and 5-FU-treated cells $(\mathrm{P}<0.05)$. Thus, these data indicate that ShCCND1 increases the apoptotic cell population and combined treatment with ShCCND1 and 5-FU synergistically increases apoptosis. Collectively, these results clearly demonstrate that the anti-proliferative effect of ShCCND1 is mediated via induction of cell cycle arrest and apoptosis.

ShCCND1 or 5-FU singly or in combination influences the expression of $p A K T, p N F \kappa B$ and downstream signal molecules. The AKT/NFKB survival signaling pathway plays a significant role in the progression of various cancers $(30,31)$. Chemoresistance to 5-FU has been reported to be caused by the induction of pAKT and pNFאB (32). Moreover, high expression of cyclin D1 protein is also associated with increased resistance of cancer cells to chemotherapeutic agents such as 5-FU $(12,13)$. Therefore, to test whether the antiproliferative effect of the combination of 5-FU and ShCCND1 is attributable to the inhibition of $\mathrm{AKT} / \mathrm{NF \kappa B}$ expression, we investigated the activation status of downstream components of $\mathrm{AKT} / \mathrm{NF} \kappa \mathrm{B}$ by western blotting. As shown in Fig. 5A, the expression of pAKT and $\mathrm{pNF} \mathrm{B}$ after 5-FU treatment was reduced, compared to that in untreated cells. Combined treatment reduced pAKT expression to a greater degree than did treatment with ShScramble $(\mathrm{P}<0.01)$, ShCCND1 $(\mathrm{P}<0.05)$, or 5 -FU $(\mathrm{P}<0.05)$ alone. In addition, combined treatment with 5-FU and ShCCND1 significantly reduced $\mathrm{pNF}$ B B expression compared to that in cells treated with ShScramble $(\mathrm{P}<0.001)$, ShCCND1 $(\mathrm{P}<0.05)$, or 5-FU $(\mathrm{P}<0.01)$ alone. These data suggest that the reduced expression of $\mathrm{pAKT}$ and $\mathrm{pNF} \kappa \mathrm{B}$ by combined treatment with ShCCND1 and 5-FU increases chemosensitivity and overcomes 5-FU resistance in AGS cells.

The expression of cyclin D1 and pRB, as measured by western blotting, was consistent with the cell cycle arrest at G1 phase, as determined by flow cytometry (Fig. 5B). The expression of cyclin D1 in cells treated with 5-FU $(\mathrm{P}<0.001)$ or ShCCND1 was reduced compared to that in untreated cells. Combined treatment with 5-FU and ShCCND1 reduced cyclin D1 expression to a greater extent compared to that in untreated cells $(\mathrm{P}<0.05)$ and in cells treated with 5 -FU or ShCCND1 alone. Analysis of pRB, a downstream molecule of cyclin D1, revealed a similar pattern. The expression level of pRB in cells treated with 5-FU or ShCCND1 was decreased compared to that in untreated cells. Combined treatment with 5-FU and ShCCND1 significantly reduced pRB levels compared to that in untreated cells $(\mathrm{P}<0.01)$ and in cells treated with ShCCND1 $(\mathrm{P}<0.05)$ or 5-FU alone.

\section{Discussion}

In spite of the availability of new chemotherapeutic treatments, the prognosis of gastric cancer is still poor $(33,34)$. 
The survival time of patients with recurrence or metastasis is $<2$ years, despite application of traditional chemotherapy (35). Moreover, the side effects of cytotoxic chemotherapeutic drugs often result in deterioration of the quality of life in patients. Accordingly, to improve the clinical outcome, more effective and innovative treatments are needed.

$5-\mathrm{FU}$ is the standard therapy against gastric cancer and is a well-known apoptosis-inducing drug that has been in use for several decades. However, resistance to 5-FU, a main cause for failure of chemotherapy, frequently develops in human gastric cancer. In previous studies, chemoresistance to 5-FU has been associated with AKT and NFKB activation and was shown to increase with increased expression of $\mathrm{pAKT}$ and pNFkB $(32,36)$. Therefore, among the numerous reported mechanisms of 5-FU resistance, in this study, we focused on the cell signaling proteins AKT and NFKB.

Cyclin D1, a cell cycle regulator, is involved in the regulation of the G1-S phase transition of the cell cycle (37). Overexpression of cyclin D1 is associated with rapid cell growth, poor prognosis and increased chemoresistance in various cancers $(25,38)$. Previous studies have distinctly shown that treatment of cancer cells with cyclin D1 antisense results in considerable inhibition of cell growth (38). Recently, silencing cyclin D1 has also been shown to induce apoptosis in some cancer cell lines $(25,39)$. To analyze the effects of silencing cyclin D1 expression on gastric cancer cell function, we first established a stable cell line expressing ShCCND1 that effectively inhibits expression of the cyclin D1 protein. As expected, the results of this study showed that cells stably transduced with ShCCND1 exhibited decreases in cyclin D1 protein levels, cell proliferation rate, cell motility and the ability to form foci. These results are in agreement with those reported in previous studies $(9,10,25)$.

If ShCCND1 can decrease the resistance of gastric cancer to 5-FU, it might be an encouraging potential anticancer therapy that can be administered along with 5-FU for treatment of gastric cancer. To review the role of ShCCND1 in regulating 5-FU sensitivity in gastric cancer, a stable cell line expressing ShScramble or ShCCND1 was used. In this study, AGS gastric cells were insensitive to 5-FU concentrations $>5 \mu \mathrm{g} / \mathrm{ml}$, whereas AGS cells treated with ShCCND1 were sensitive to lower concentrations of 5-FU. Combined treatment with ShCCND1 and 5-FU was more effective than treatment with either agent alone. As expected, cell proliferation was inhibited, G1 arrest was enhanced and apoptosis was induced in AGS cells exposed to ShCCND1 or 5-FU alone or in combination. These results demonstrate that the expression level of cyclin D1 is related to the chemosensitivity to 5-FU and that chemosensitivity might be generated or restored by decreasing the expression of cyclin D1.

The AKT and NFKB proteins play important roles in cancer progression, including cell proliferation, cell invasion, apoptosis and metastasis (30). Furthermore, their activation is also associated with chemosensitivity to 5-FU (40). In a previous report, chemoresistance to $5-\mathrm{FU}$ was shown to be caused by an increase in pAKT and pNFKB expression (32). Moreover, overexpression of cyclin D1 protein also increases resistance of cancer cells to chemotherapeutic agents such as 5-FU $(12,13)$. In this study, combined treatment with ShCCND1 and 5-FU suppressed the expression of pAKT,
pNFKB and cyclin D1 proteins to a greater extent than that in cells treated with ShCCND1 or 5-FU alone. In addition, the expression of $\mathrm{pRB}$, which is directly regulated by cyclin $\mathrm{D} 1$, was significantly downregulated in cells that received the combined treatment than in those that received the single treatment. These results support the notion that the 5-FU and ShCCND1 combination exerts a synergistic killing effect in human gastric cancer cells and that this effect is attributable to the suppression of $\mathrm{pAKT}$ and $\mathrm{pNF} \kappa \mathrm{B}$ expression. Taken together, the results of this study provide further evidence that therapeutic strategies targeting cyclin D1 may have the dual advantage of suppressing the growth of cancer cells, while concomitantly enhancing their chemosensitivity.

\section{Acknowledgements}

This study was supported by Konkuk University in 2011.

\section{References}

1. Leung WK, Wu MS, Kakugawa Y, Kim JJ, Yeoh KG, Goh KL, Wu KC, Wu DC, Sollano J, Kachintorn U, Gotoda T, Lin JT, You WC, Ng EK, Sung JJ and Asia Pacific Working Group on Gastric C: Screening for gastric cancer in Asia: current evidence and practice. Lancet Oncol 9: 279-287, 2008.

2. Ohtsu A: Chemotherapy for metastatic gastric cancer: past, present and future. J Gastroenterol 43: 256-264, 2008.

3. Sakuramoto S, Sasako M, Yamaguchi T, Kinoshita T, Fujii M, Nashimoto A, Furukawa H, Nakajima T, Ohashi Y, Imamura H, Higashino M, Yamamura Y, Kurita A and Arai K: Adjuvant chemotherapy for gastric cancer with S-1, an oral fluoropyrimidine. N Engl J Med 357: 1810-1820, 2007.

4. GASTRIC (Global Advanced/Adjuvant Stomach Tumor Research International Collaboration) Group, Paoletti X, Oba K, Burzykowski T, Michiels S, Ohashi Y, Pignon JP, Rougier P, Sakamoto J, Sargent D, Sasako M, Van Cutsem E and Buyse M: Benefit of adjuvant chemotherapy for resectable gastric cancer: a meta-analysis. JAMA 303: 1729-1737, 2010.

5. Sherr CJ: G1 phase progression: cycling on cue. Cell 79: 551-555, 1994.

6. Weinberg RA: The retinoblastoma protein and cell cycle control. Cell 81: 323-330, 1995.

7. Gao P, Zhou GY, Liu Y, Li JS, Zhen JH and Yuan YP: Alteration of cyclin D1 in gastric carcinoma and its clinicopathologic significance. World J Gastroenterol 10: 2936-2939, 2004.

8. Motokura T and Arnold A: Cyclin D and oncogenesis. Curr Opin Genet Dev 3: 5-10, 1993.

9. Coco Martin JM, Balkenende A, Verschoor T, Lallemand F and Michalides R: Cyclin D1 overexpression enhances radiationinduced apoptosis and radiosensitivity in a breast tumor cell line. Cancer Res 59: 1134-1140, 1999.

10. Han EK, Begemann M, Sgambato A, Soh JW, Doki Y, Xing WQ, Liu W and Weinstein IB: Increased expression of cyclin D1 in a murine mammary epithelial cell line induces p27kip1, inhibits growth and enhances apoptosis. Cell Growth Differ 7: 699-710, 1996.

11. Kuroda Y, Sakai A, Tsuyama N, Katayama Y, Munemasa S, Asaoku H, Okikawa Y, Nakaju N, Mizuno M, Ogawa K, Nishisaka T, Matsui $\mathrm{H}$, Tanaka $\mathrm{H}$ and Kimura A: Ectopic cyclin D1 overexpression increases chemosensitivity but not cell proliferation in multiple myeloma. Int J Oncol 33: 1201-1213, 2008.

12. Kornmann M, Arber N and Korc M: Inhibition of basal and mitogen-stimulated pancreatic cancer cell growth by cyclin D1 antisense is associated with loss of tumorigenicity and potentiation of cytotoxicity to cisplatinum. J Clin Invest 101: 344-352, 1998.

13. Warenius HM, Seabra LA and Maw P: Sensitivity to cisdiamminedichloroplatinum in human cancer cells is related to expression of cyclin D1 but not C-RAF-1 protein. Int J Cancer 67: 224-231, 1996

14. Simizu S, Shibasaki F and Osada H: Bcl-2 inhibits calcineurinmediated Fas ligand expression in antitumor drug-treated baby hamster kidney cells. Jpn J Cancer Res 91: 706-714, 2000. 
15. Zhou X, Zhang Z, Yang X, Chen W and Zhang P: Inhibition of cyclin D1 expression by cyclin D1 shRNAs in human oral squamous cell carcinoma cells is associated with increased cisplatin chemosensitivity. Int J Cancer 124: 483-489, 2009.

16. Sun Y, Luo D and Liao DJ: Cyclin D1 protein plays different roles in modulating chemoresponses in MCF7 and MDA-MB231 cells. J Carcinog 11: 12, 2012.

17. Cardone MH, Roy N, Stennicke HR, Salvesen GS, Franke TF, Stanbridge E, Frisch S and Reed JC: Regulation of cell death protease caspase-9 by phosphorylation. Science 282: 1318-1321, 1998.

18. Brunet A, Bonni A, Zigmond MJ, Lin MZ, Juo P, Hu LS, Anderson MJ, Arden KC, Blenis J and Greenberg ME: Akt promotes cell survival by phosphorylating and inhibiting a Forkhead transcription factor. Cell 96: 857-868, 1999.

19. Curnock AP, Logan MK and Ward SG: Chemokine signalling: pivoting around multiple phosphoinositide 3-kinases. Immunology 105: 125-136, 2002.

20. Baldwin AS: Control of oncogenesis and cancer therapy resistance by the transcription factor NF-kappaB. J Clin Invest 107 241-246, 2001.

21. Ozes ON, Mayo LD, Gustin JA, Pfeffer SR, Pfeffer LM and Donner DB: NF-kappaB activation by tumour necrosis factor requires the Akt serine-threonine kinase. Nature 401: 82-85, 1999.

22. Wang C-Y, Mayo MW and Baldwin AS: TNF- and cancer therapy-induced apoptosis: potentiation by inhibition of NF-kappaB. Science 274: 784-787, 1996.

23. Camp ER, Li J, Minnich DJ, Brank A, Moldawer LL, MacKay SL and Hochwald SN: Inducible nuclear factor-kappaB activation contributes to chemotherapy resistance in gastric cancer. J Am Coll Surg 199: 249-258, 2004.

24. Lu YS, Yeh PY, Chuang SE, Gao M, Kuo ML and Cheng AL: Glucocorticoids enhance cytotoxicity of cisplatin via suppression of $\mathrm{NF}-\kappa \mathrm{B}$ activation in the glucocorticoid receptor-rich human cervical carcinoma cell line SiHa. J Endocrinol 188: 311-319, 2006.

25. Sauter ER, Nesbit M, Litwin S, Klein-Szanto AJ, Cheffetz S and Herlyn M: Antisense cyclin D1 induces apoptosis and tumor shrinkage in human squamous carcinomas. Cancer Res 59: 4876-4881, 1999.

26. Huang WS, Wang JP, Wang T, Fang JY, Lan P and Ma JP: ShRNA-mediated gene silencing of beta-catenin inhibits growth of human colon cancer cells. World J Gastroenterol 13 6581-6587, 2007

27. Liang CC, Park AY and Guan JL: In vitro scratch assay: a convenient and inexpensive method for analysis of cell migration in vitro. Nat Protoc 2: 329-333, 2007.

28. Tiang JM, Butcher NJ and Minchin RF: Small molecule inhibition of arylamine $\mathrm{N}$-acetyltransferase Type I inhibits proliferation and invasiveness of MDA-MB-231 breast cancer cells. Biochem Biophys Res Commun 393: 95-100, 2010.
29. Pearson G, English JM, White MA and Cobb MH: ERK5 and ERK2 cooperate to regulate NF-kappaB and cell transformation. J Biol Chem 276: 7927-7931, 2001.

30. Martin V, Herrera F, Carrera-Gonzalez P, Garcia-Santos G, Antolin I, Rodriguez-Blanco J and Rodriguez C: Intracellular signaling pathways involved in the cell growth inhibition of glioma cells by melatonin. Cancer Res 66: 1081-1088, 2006.

31. Cheng JC, Chou CH, Kuo ML and Hsieh CY: Radiationenhanced hepatocellular carcinoma cell invasion with MMP-9 expression through PI3K/Akt/NF-kappaB signal transduction pathway. Oncogene 25: 7009-7018, 2006.

32. Shin JY, Kim JO, Lee SK, Chae HS and Kang JH: LY294002 may overcome 5-FU resistance via down-regulation of activated p-AKT in Epstein-Barr virus-positive gastric cancer cells. BMC Cancer 10: 425, 2010.

33. Kim TW, Kang YK, Ahn JH, Chang HM, Yook JH, Oh ST, Kim BS and Lee JS: Phase II study of capecitabine plus cisplatin as first-line chemotherapy in advanced gastric cancer. Ann Oncol 13: 1893-1898, 2002

34. Song M, Zhang R, Dai Y, Gao F, Chi H, Lv G, Chen B and Wang $X$ : The in vitro inhibition of multidrug resistance by combined nanoparticulate titanium dioxide and UV irradition. Biomaterials 27: 4230-4238, 2006.

35. Yoshida K and Toge T: Combination chemotherapy of TS-1 and docetaxel on advanced and recurrent gastric cancer. Gan To Kagaku Ryoho 31: 1982-1986, 2004.

36. Arlt A, Gehrz A, Muerkoster S, Vorndamm J, Kruse ML, Folsch UR and Schafer H: Role of NF-kappaB and Akt/PI3K in the resistance of pancreatic carcinoma cell lines against gemcitabine-induced cell death. Oncogene 22: 3243-3251, 2003.

37. Fu M, Wang C, Li Z, Sakamaki T and Pestell RG: Minireview: Cyclin D1: normal and abnormal functions. Endocrinology 145 : 5439-5447, 2004.

38. Kornmann M, Danenberg KD, Arber N, Beger HG, Danenberg PV and Korc M: Inhibition of cyclin D1 expression in human pancreatic cancer cells is associated with increased chemosensitivity and decreased expression of multiple chemoresistance genes. Cancer Res 59: 3505-3511, 1999.

39. Zhou H, Fujigaki Y, Kato A, Miyaji T, Yasuda H, Tsuji T, Yamamoto T, Yonemura $\mathrm{K}$ and Hishida A: Inhibition of p21 modifies the response of cortical proximal tubules to cisplatin in rats. Am J Physiol Renal Physiol 291: F225-F235, 2006.

40. Liu N, Zhang J, Zhang J, Liu S, Liu Y and Zheng D: Erbinregulated sensitivity of MCF-7 breast cancer cells to TRAIL via ErbB2/AKT/NF-kappaB pathway. J Biochem 143: 793-801, 2008. 